\title{
E-Books und E-Book-Pakete
}

\section{E-Books}

Obwohl ihr Marktanteil noch bei nur wenigen Prozenten liegt, sind E-Books derzeitig sowohl für den Buchhandel als auch für die Bibliotheken ein viel diskutiertes Thema. Im Angebot von Universitätsund Hochschulbibliotheken, aber auch von Staatsund Landesbibliotheken sind E-Books mittlerweile fest verankert - so auch in der Württembergischen Landesbibliothek. Schon seit Längerem bietet sie ihren registrierten Benutzern E-Books im Rahmen lizenzpflichtiger Datenbanken an. Die E-Books sind im Online-Katalog nachgewiesen und mit einem internetfähigen Computer entweder in den Räumen der Bibliothek oder von außerhalb, d. h. mit Remote Access, zu benutzen.

\section{Zu diesen E-Books gehören z. B. Werkausgaben} von Bertolt Brecht, Johann Wolfgang von Goethe, Franz Kafka, Martin Luther und Friedrich Schiller sowie die Deutsche Literatur des 18. Jahrhunderts Online, die alle mit Remote Access benutzbar sind. Dazu gehören ebenso E-Books aus den Nationallizenzen Springer eBooks Chemistry \& Material Science und Springer eBooks Medicine, die einzeln im Online-Katalog recherchierbar und ebenfalls mit Remote Access benutzbar sind. Und es gehören die E-Books aus den Datenbanken WISO und Beck online dazu, die in der Benutzung einen hohen Stellenwert haben. Auch die E-Books aus WISO und Beck online sind einzeln im Online-Katalog nachgewiesen, wobei die E-Books aus WISO mit Remote Access und die aus Beck online aus lizenzrechtlichen Gründen leider nur mit einem InhouseZugriff angeboten werden dürfen, d. h. letztere sind gemäß Vorgabe des Verlags Beck lediglich in den Räumen der Bibliothek benutzbar.

Um das E-Book-Angebot relativ kurzfristig merklich zu erhöhen und auf mehr Wissenschaftsfächer auszudehnen, entschied man sich 2012 für die Erwerbung von E-Book-Paketen. Ein wissenschaftlicher Verlag fasst in der Regel seine E-Books pro Erscheinungsjahr zu Paketen zusammen. Ein Paket enthält, abhängig von der Größe und vom Profil des Verlags, die E-Books eines Wissenschaftsfachs, mehrerer verwandter Fächer oder die Gesamtpro- duktion eines Jahres. Eine Bibliothek erwirbt eines oder mehrere der Pakete.

Für die Württembergische Landesbibliothek mussten als erstes Auswahlkriterien für die Paketerwerbung entwickelt werden. Danach waren Erwerbungs- und Erschließungsfragen zu klären. Ein Geschäftsgang musste aufgebaut werden, wobei der Nachweis des einzelnen E-Books im OnlineKatalog als notwendig angesehen wurde. Die Präsentation von E-Book-Paketen in DBIS war zu erarbeiten. Und schließlich mussten für die Website das Angebot und die Nutzungsbedingungen von E-Books formuliert werden.

\section{E-Book-Pakete}

\section{a) Auswahl, Kauf und Lizenzierung}

Die Württembergische Landesbibliothek ist eine wissenschaftliche Universalbibliothek mit Schwerpunkt auf den Geistes- und Sozialwissenschaften. Die Auswahl von E-Book-Paketen erfolgt darum mit einer geistes- und sozialwissenschaftlichen Ausrichtung.

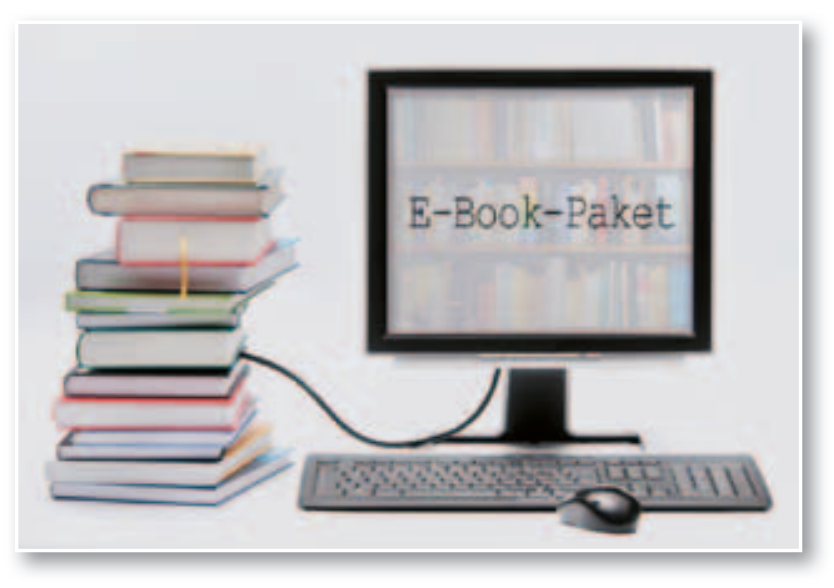

Informationen über konsortiale Angebote wurden eingeholt, und parallel wurden mit Buchhändlern Verhandlungen über Preise und Konditionen geführt. Die Paketerwerbung durch einen Buchhändler erhielt schließlich den Vorzug, um den Buchhandelsservice für die Preisermittlung, Rechnungsstellung, Metadatenlieferung, Zugangs-URL, Zugriffsrechte und gegebenenfalls Reklamationen in Anspruch zu nehmen. 
Im Hinblick auf die Benutzung wurden folgende Bedingungen für die Paketerwerbung formuliert:

- Es werden ausschließlich E-Book-Pakete solcher Verlage ausgewählt, die den Remote Access zulassen.

- Die Authentifizierung registrierter Bibliotheksbenutzer für den Remote Access erfolgt wie bei Datenbanken und E-Journals über den HANServer.

- Das Digital Rights Management (DRM) sollte möglichst moderat ausfallen.

Nicht in Betracht kamen Verlage, die für eine Landesbibliothek keinen Remote Access zulassen, z. B. die Springer-Imprints Gabler und VS. Ebenso wurde zunächst auf die Verlage verzichtet, die ihre E-Books ausschließlich über Aggregatoren anbieten, z. B. Vandenhoeck und Ruprecht oder Meiner. Generell wurde auf die Einbeziehung von Verlagen aus Baden-Württemberg verzichtet; deren E-Books sollen separat über die Pflichtablieferung erworben werden.

Last but not least ist die Erwerbung von E-Book-Paketen auch eine Preisfrage. Am Ende des Auswahlprozesses wurden nicht nur die Verlagsangebote angenommen, die vom Profil her passen und die für eine Landesbibliothek den Remote Access erlauben, sondern die aus vorhandenen Erwerbungsmitteln auch finanzierbar waren.

Um den Benutzern gleich zu Beginn der Paketerwerbung eine hohe Zahl an E-Books anzubieten, wurden E-Books mit den Erscheinungsjahren 2010, 2011 und 2012 erworben. Die Entscheidung wurde getroffen für Hanser (die Fachpakete Wirtschaft und Qualitätsmanagement), Campus (die Gesamtpakete), Duncker \& Humblot (die Gesamtpakete) sowie UTB (das aktuelle Gesamtpaket). Während die E-Book-Pakete von Hanser, Campus und Duncker \& Humblot käuflich erworben wurden, hat man die E-Books von UTB über das sog. Gebührenmodell lizenziert; denn nur für dieses Geschäftsmodell erlaubt UTB den Remote Access - leider mit einem sehr restriktiven DRM.

\section{b) Präsentation im Online-Katalog und in DBIS}

Der Online-Katalog der Württembergischen Landesbibliothek ist das zentrale Verzeichnis der Bibliotheksbestände. Oder aus der Sicht der Benut- zung kann man sagen: Der Online-Katalog ist das zentrale Findmittel der Bibliothek. Jedes einzelne lizenzierte E-Book, egal ob es als Teil einer Datenbank oder eines E-Book-Paketes zum Bestand der Bibliothek gehört, soll über den Online-Katalog recherchierbar sein. Wie jedes gedruckte Buch wird darum auch jedes E-Book in der Katalogisierungsdatenbank erfasst.

Zu Beginn der E-Book-Paketerwerbung ging die Abteilungsleitung der Medienbearbeitung davon aus, dass die manuelle Katalogisierung von E-Books aus Paketen nicht erforderlich sein würde. Vielmehr war daran gedacht, die bibliographischen Metadaten der Anbieter oder bereits vorhandene Metadaten aus anderen Bibliotheksverbünden zu nutzen, d. h. vom BSZ die Metadaten in die Verbunddatenbank einspielen zu lassen, automatisiert Lokalsätze erzeugen zu lassen und die Daten dann für den hauseigenen Online-Katalog zu übernehmen. Bei einzelnen Verlagspaketen hat es gut funktioniert, automatisiert Lokalsätze zu erzeugen. Schwieriger als erwartet, hat sich die Möglichkeit zur maschinellen Datenübernahme aus anderen Bibliotheksverbünden herausgestellt. Leider kann eben doch nicht durchweg darauf verzichtet werden, manuell zu katalogisieren. Der Personalaufwand für die Erwerbung und Katalogisierung von E-Books wird dadurch höher als zunächst angenommen.

Jedes E-Book-Paket wird im Online-Katalog nachgewiesen. Zusätzlich wird das Zugriffsrecht angezeigt, und der Link zu DBIS wird angegeben:

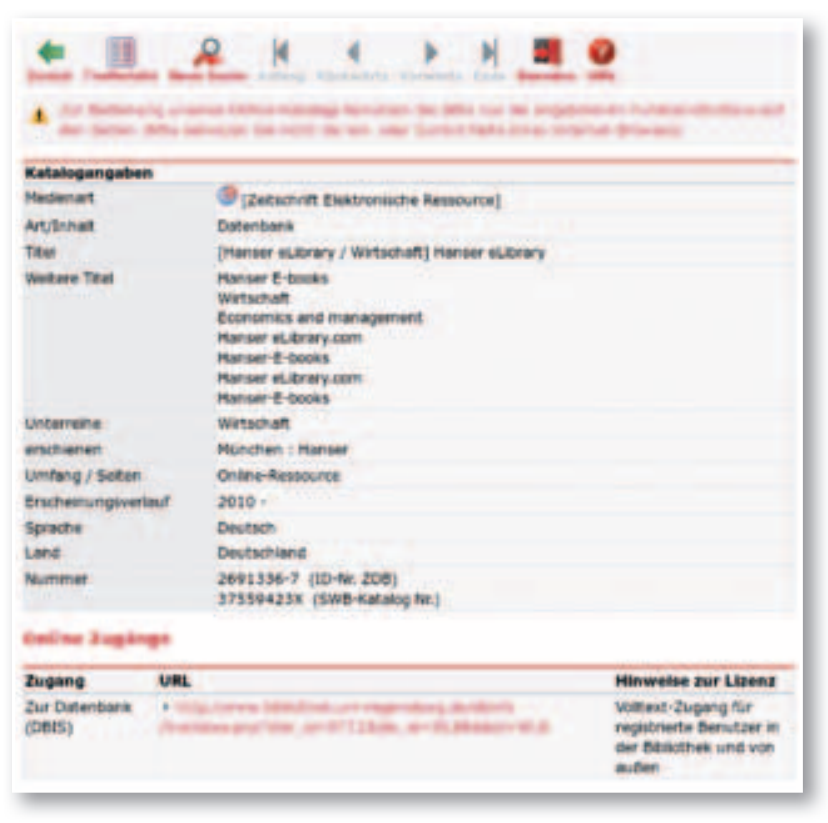


Die Anzeige der E-Book-Liste eines Verlages sieht im Online-Katalog folgendermaßen aus:

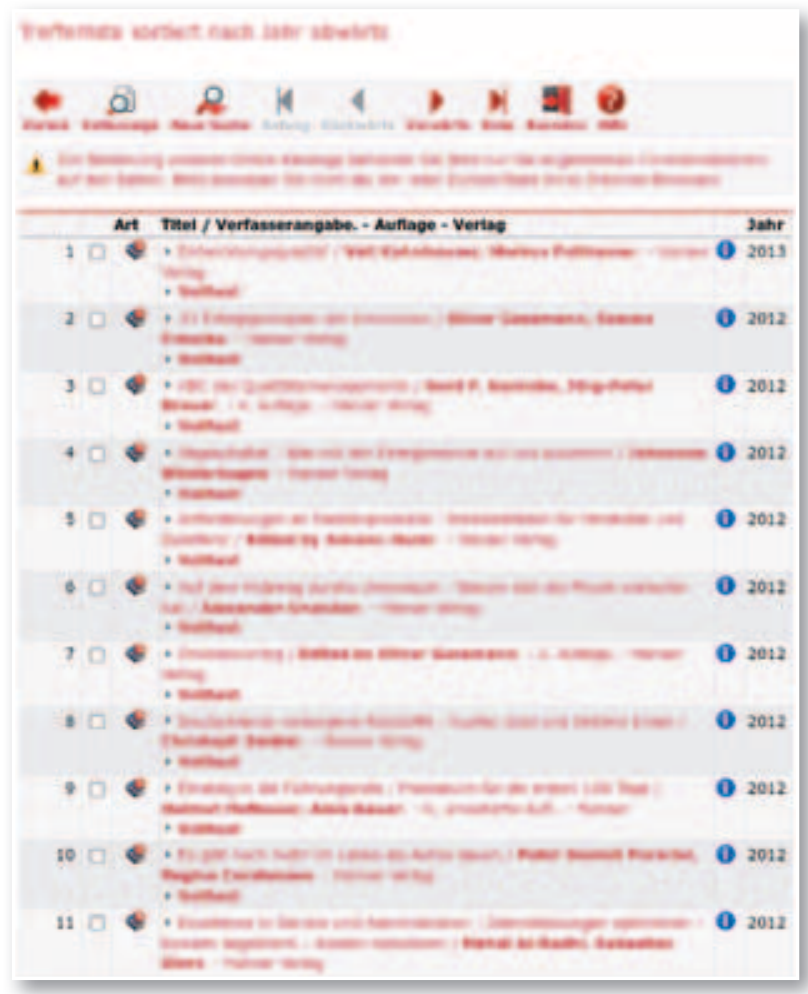

Die Vollanzeige für ein einzelnes E-Book inkl. der Angabe des Zugriffsrechts sieht so aus:

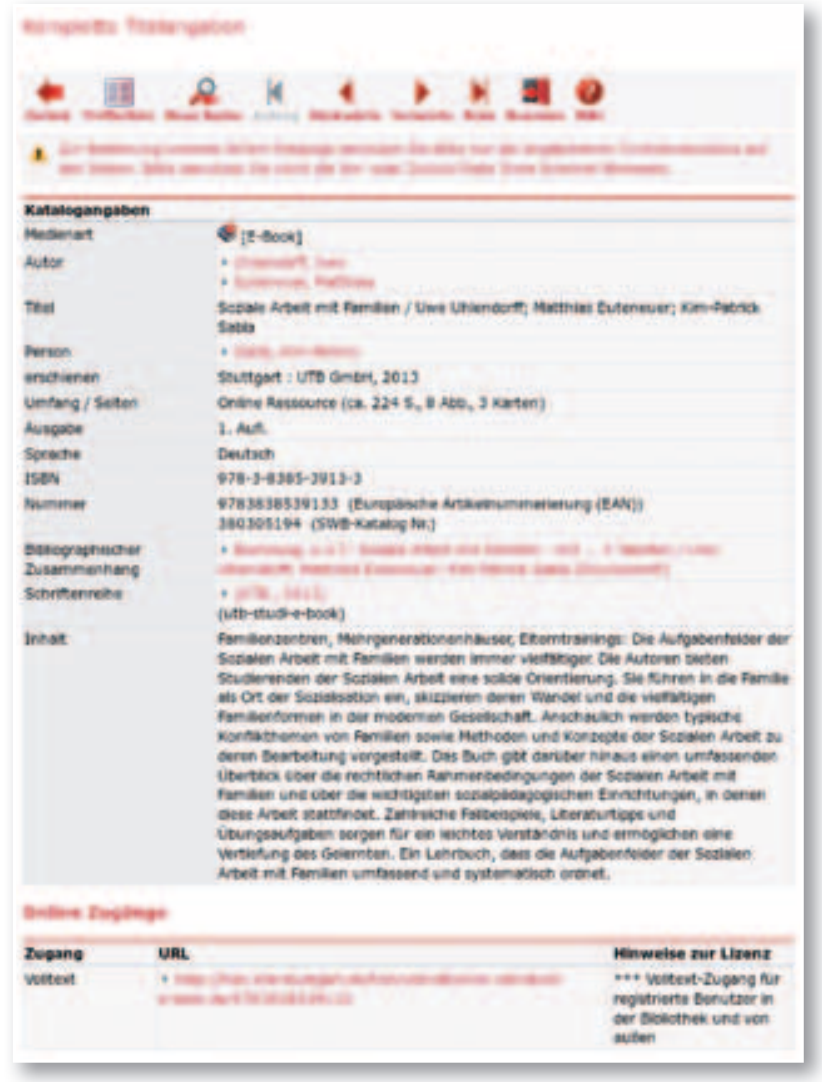

Neben den Nachweis im Online-Katalog tritt bei EBook-Paketen der Nachweis in DBIS. Dazu wurde in DBIS eigens die "Sammlung: E-Books" angelegt. Diese listet außer den E-Book-Paketen die lizenzpflichtigen Datenbanken und die Nationallizenzen mit E-Books auf. Freie E-Book-Sammlungen werden in Auswahl berücksichtigt. Die Präsentation in DBIS stellt damit die E-Book-Sammlungen heraus, die die Bibliothek ihrem Profil entsprechend erworben und finanziert hat. Die Anzeige freier E-Book-Sammlungen tritt hinter der Anzeige lizenzierter Bestände zurück. Als TOP-Datenbanken werden die Angebote aufgelistet, deren E-Books einzeln im Online-Katalog nachgewiesen sind.

\section{E-Book-Benutzung}

Die Württembergische Landesbibliothek bietet inzwischen ca. 16.000 lizenzierte E-Books zur Benutzung an. Der Benutzer findet diese E-Books ohne besondere Rechercheanleitung im OnlineKatalog. Unter Umständen findet die Verzeichnung neuer Pakete im Online-Katalog mit einer gewissen zeitlichen Verzögerung statt. Einen Überblick über die vorhandenen E-Book-Sammlungen und deren Benutzung erhält man in DBIS.

Für die Benutzung ist von grundsätzlicher Bedeutung, dass die lizenzierten E-Books den registrierten persönlichen Bibliotheksbenutzern vorbehalten sind. Um ein E-Book aufrufen zu können, muss man sich mit seiner Benutzernummer und seinem Passwort als Benutzer der WLB authentifizieren. Dafür setzt die WLB den HAN-Server der Firma $\mathrm{H}+\mathrm{H}$ ein, der per IP-Kontrolle Zugang zum Verlagsserver erhält. Die Authentifizierung und Autorisierung mit Shibboleth setzen erst wenige E-BookAnbieter ein.

Die Nutzung von E-Books ist den Benutzern ausschließlich für den eigenen wissenschaftlichen und privaten Gebrauch gestattet. Jede Art der Nutzung zu gewerblichen Zwecken ist untersagt. Eine institutionelle Nutzung ist nicht zulässig.

Die vom E-Book-Anbieter vorgegebene Lizenz regelt, ob das E-Book ausschließlich in den Räumen der Bibliothek oder auch von außen zugänglich ist. Ebenso bestimmt der Anbieter das Digital Rights Management (DRM), wodurch der Umfang von Ausdruck, Download und Kopie festgelegt wird. 


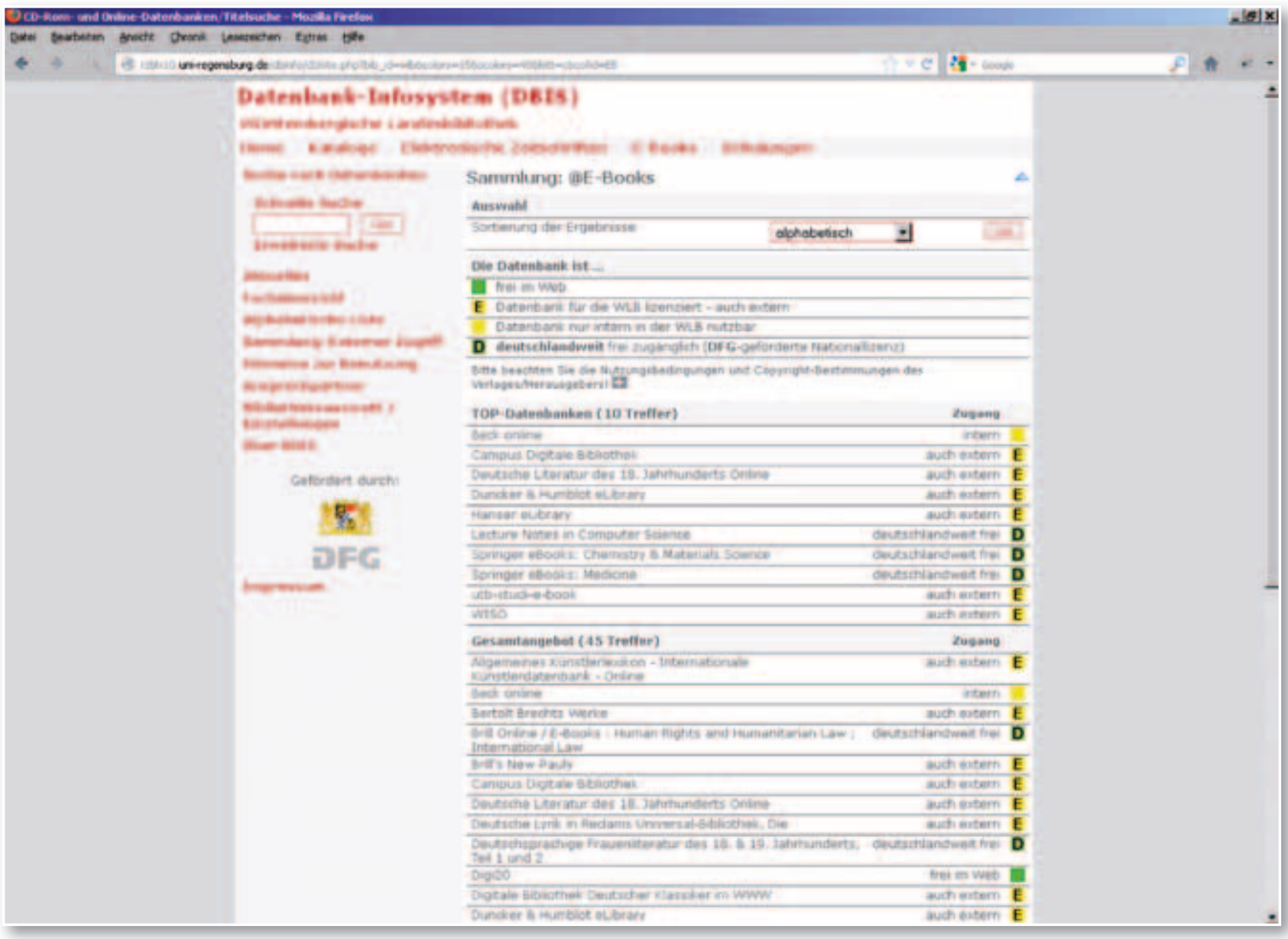

Sammlung E-Books in DBIS

An die neuartigen Nutzungsbedingungen eines EBooks im Vergleich zum gedruckten Buch müssen sich die Benutzer und auch die Bibliothekare zunächst einmal gewöhnen. Dann allerdings sind die Vorteile, die die E-Books in der Benutzung mit sich bringen, schnell zu erkennen:

- E-Books können rund um die Uhr von mehreren Benutzern gleichzeitig genutzt werden.

- Der Großteil der E-Books wird mit Remote Access angeboten, wodurch eine Benutzung von zu Hause aus oder von jedem anderen externen Standort aus möglich ist.

- Für E-Books entfallen Wartezeiten nach der Bestellung.

- Für E-Books entfallen die Vormerkungen.
- Die Texte der E-Books können am Bildschirm nicht nur gelesen, sondern auch durchsucht und bearbeitet werden.

- Teile eines E-Books können ausgedruckt oder zur Weiterbearbeitung abgespeichert werden.

- E-Books verfügen häufig über Zusatzfunktionen wie eine Markier- oder Kopierfunktion für die Zitatentnahme.

- E-Book-Daten lassen sich in Literaturverwaltungssystemen unkompliziert weiterverarbeiten.

Erste Auswertungen von Benutzungszahlen weisen darauf hin, dass die E-Books von den Benutzern angenommen werden. 2013 werden die Paketerwerbungen fortgesetzt, auch um die Benutzung breiter evaluieren zu können.

\section{Angelika Schütt-Hohenstein}

